\title{
High precision scanning system and test technology of remote sensor
}

\author{
Yang Xiaole, Kang Jianbing, Wang Dongjie and Zhao Xin \\ Beijing institute of space Mechanics \& Electricity china
}

\begin{abstract}
Reliability of scanning system is more important for scanning remote sensor. The sensor also has a higher request for angle measuring precision, control precision and non-linearity as the raise of resolution and $\mathrm{S} / \mathrm{N}$. A new high reliability and precision scanning system are designed. Choosing for shafting, motor and angle measuring sensor, scanning control circuit design and control scheme are introduced. High precision system needs high precision test system, a high precision non-contact test system is introduced finally. The result of test shows that angle measuring precision is smaller than 1 arcsecond and non-linearity is less than $0.5 \%$.
\end{abstract}

\section{Introduction}

Scanning system which drives and controls scanning mirror to swing linearly through the vertical flight direction is the key system of scanning optical remote sensor. The scanning system consists of scanning mirror, bearing, motor, angle sensor and control circuit etc. Scanning system is usually evaluated by scanning non-linearity, control precision, angle measuring precision, scanning efficiency and so on. Scanning system is a typical mechanical and electrical system, whose precision is decided by mechanical system and electronic control. With the increasing of spatial resolution and aperture, scanning mirror size and moment of inertia also increase, so control difficulty increases. This paper introduces high precision and high reliability scanning system based on the beryllium mirror, flexible pivot, inductosyn and voice coil motor in detail from two aspects ${ }^{[1]}$.

\section{Scanning mirror}

Scanning mirror ensures strength and rigidity of the mirror body, meanwhile, the mass and moment of inertia is minimized, so it can reduce influence on support element life and disturbing torque to the satellite platform. Scanning mirror size determined by the aperture of optical system is $600 \mathrm{~mm} \times 400 \mathrm{~mm}$, so low density, high specific rigidity, high specific strength and thermal stability materials is chose to use as mirror substrate. Beryllium is recognized as the best material, and $\mathrm{SiC}$ is also commonly materials ${ }^{[1]}$. Beryllium is light than $\mathrm{SiC}$ but toxic, and still need lightweight. Scanning mirror can reduced weighing by $65 \%$, only $6 \mathrm{~kg}$ through lightweight. Corresponding moment of inertia is only $0.13 \mathrm{~kg} . \mathrm{m}^{2}$.

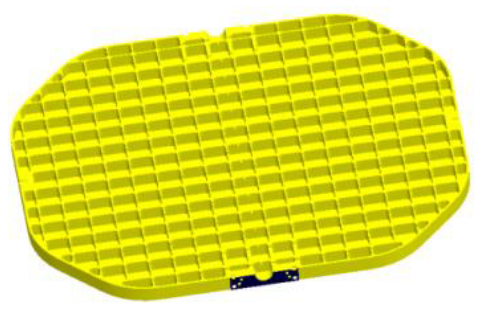

Figure 1. Configuration diagram of scanning mirror

\section{Scanning shafting}

The scanning shafting which is the key component provides support for scanning mirror, and the support rigidity and precision directly affect system life and control precision.

Bearing is used commonly, but oil lubricated bearings used in space application has the problem of vacuum cold welding and the harm of optical mirror pollution. It needs to use ultra low volatile oil and seal structure, and the reliability and long life is a difficult problem. Solid lubricated rolling bearing may decreases precision or has deadlocking problem due to friction when working for a long time ${ }^{[1]}$.

Flexible pivot used in this paper is frictionless and elastic support, and achieving relative rotation relies on elastic material deformation around a fixed axis. No metal to metal contact, only intermolecular friction, so friction moment is lowest. Pivot structure is showed in figure 2, and it consists of three cross tablet spring (narrow tablet spring I, II and wide tablet spring) and two identical cylindrical hollow tablet spring seats. The spring and spring seat use welding technology.

Keeping flexible pivot in position needs torque motor, because of restoring moment ${ }^{[2]}$. 
Restoring moment coefficient of flexible pivot is $1.5025 \mathrm{~N} \cdot \mathrm{m} / \mathrm{rad}$, maximum restoring moment is $0.132 \mathrm{~N} . \mathrm{m}$ accords to maximum angle. Each shafting uses two flexible pivots, so the maximum restoring moment is $0.264 \mathrm{~N} \cdot \mathrm{m}$. In the practical application, we should be as far as possible to filter out two pivot with the same restoring moment coefficient, meanwhile, motor and angle measuring sensor are respectively connected with two pivot, so as to avoid motor interfering sensor.

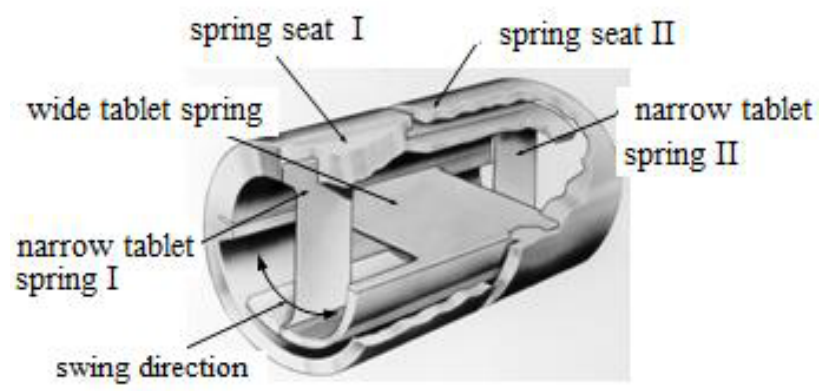

Figure 2. Structure diagram of flexible pivot

The main factor affecting scanning mechanism life is shafting life, according to using state, pivot is mainly affected by radial force during ground debugging, at the same time, radial force caused by nonaxiality should be considered. Pivot radial load ratio is calculated, according to pivot life curve in figure3, pivot (ground debugging + orbit application) is infinite life in the whole life cycle, the abscissa is load ratio, longitudinal coordinates is torsion angle.

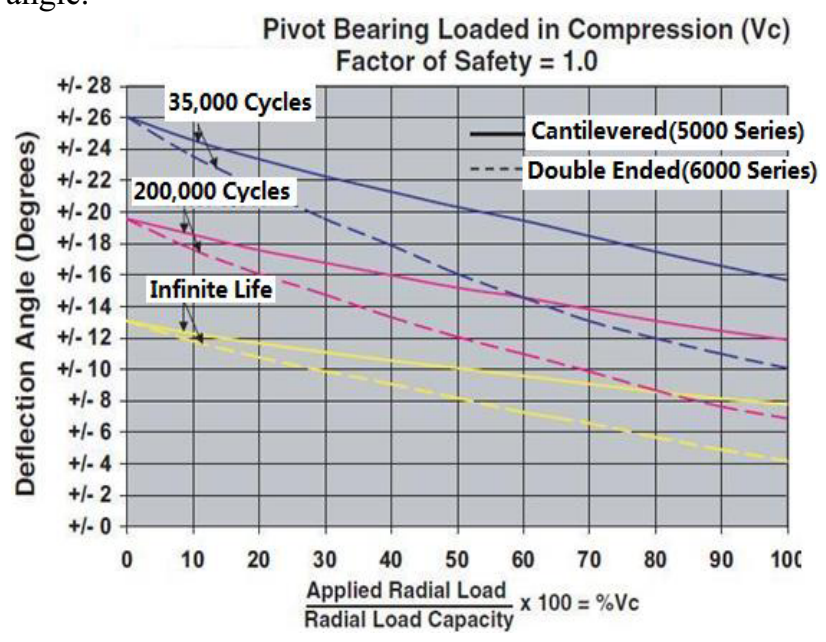

Figure 3. Pivot life curve

\section{Motor}

Scanning mirror is driven directly, this means it is directly connected to rotating axis. Motor is required to swing at a constant speed, so the requirements of the motor are ${ }^{[3]}$ :

(1) Small moment ripple;

(2) Linearity of the torque current characteristic is better;

(3) Small moment of inertia, quick dynamic response;

(4) Big moment;

(5) Long life.
Scanning mirror is driven by voice coil motor, which is a special motor and has the characteristic of simple structure, small size, high speed, fast response and high reliability etc. Voice coil motor is considered to be almost perfect servo drive device, and exercise frequency is $500-1000 \mathrm{~Hz}$ or even higher. Its working is based on principle of ampere force. The simplest linear voice coil motor is a tubular coil winding located in radial electromagnetic field. Bending two ends of linear voice coil motor into arc, and it will become rotary voice coil motor $^{[4]}$

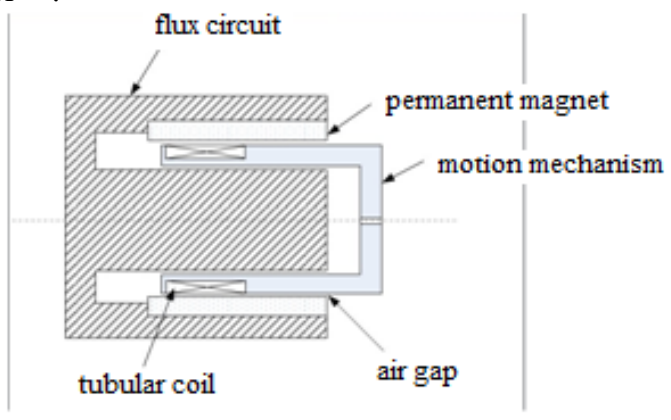

Figure 4. Structure diagram of voice coil motor

The voice coil motor focus on index of continuous torque, peak torque, current torque coefficient, angle range and weight etc.

The maximum torque requirement in this project is $0.15 \mathrm{~N} \cdot \mathrm{m}$, in the linear and nonlinear junction, continuous torque of motor is $0.64 \mathrm{~N} \cdot \mathrm{m}$, so safety factor is 4.3 and safety margin is 3.3 .

The temperature of the coil should be strictly controlled, because increasing temperature will change motor output torque.

\section{Sensors}

The sensor used in servo control system is mainly photoelectric encoder, inductosyn and resolver etc. Photoelectric encoder has large weight and volume, and ability of anti vibration and shock is weak. Principle of inductosyn and resolver is basically same. Requirement of installation precision is relatively low and the precision and repeat performance is not affected by temperature. Inductosyn has higher precision, but has higher requirement for signal processing circuit due to weaker out signal ${ }^{[5]}$. In this project, swing angle range is $10^{\circ}$, absolutely angle measuring precision is better than \pm 1 ", so Absolute 360 pole inductosyn whose angle measuring precision is \pm 1 " between $60^{\circ}$ is used. The wire is leaded from rotor, and plate is $8 \mathrm{~mm}$ thick aluminum with diameter of $130 \mathrm{~mm}$. Mechanical installation needs to ensure stator and rotor parallelism is better than 0.03 , coaxial degree is better than $\phi 0.03$.

\section{Scanning control system}

The mechanical structure which consists of beryllium mirror, voice coil motor, flexible pivot and inductosyn guarantee the system has minimum moment of inertia, minimal disturbing torque and highest angle measuring 
precision. Scanning control system makes scanning mechanism swing stably and accurately according to the given speed. In this paper, scanning control system is divided into angle measuring circuit and control circuit. Because output signal of inductosyn is only millivolt level, so scanning angle measuring circuit is put as much as possible near inductosyn to reduce signal transmission distance. Angle information is transmitted to control circuit after converting into digital quantity.

\subsection{Scanning angle measuring circuit ${ }^{[6]}$}

Angle measuring circuit based on absolute inductosyn consists of excitation module, signal conditioning module, axis angle digital conversion module, data processing module and interface module and so on, and takes FPGA as the system core.

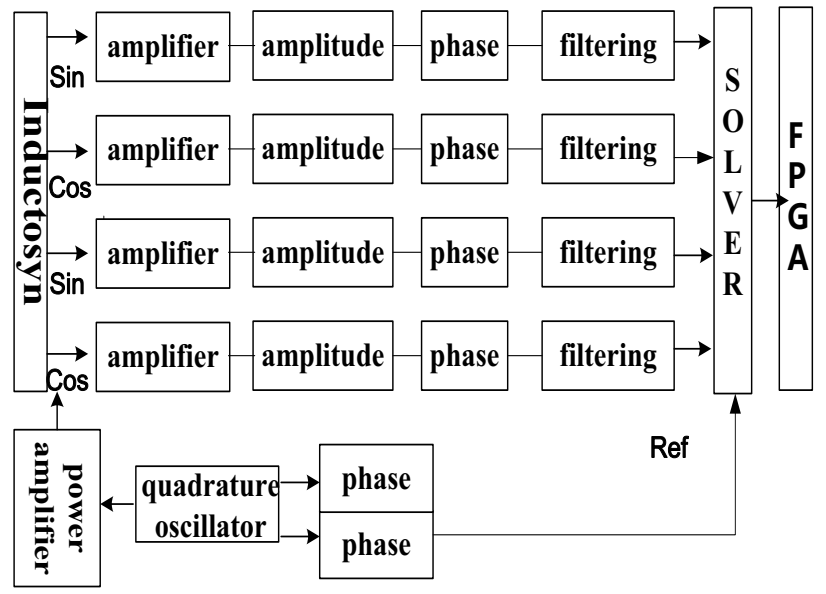

Figure 5. Principle diagram of angle measurement circuit

\subsubsection{Exciting circuit}

Excitation circuit selects dual channel series excitation mode, and uses quadrature oscillator as oscillation source. In order to increase driving capability, power amplifier is added behind oscillator; otherwise it will appear distortion waveform. It is best to use shielded twisted pair cable to reduce the interference of excitation signal. The excitation frequency is $10 \mathrm{KHz}$.

\subsubsection{Signal conditioning circuit}

Signal conditioning circuit consists of preamplifier, main amplifier, amplitude modulation, phase modulation and filtering. In order not to affect angle measuring precision, signal conditioning circuit noise must meet noise demand of axial angle solver chip AD2S80. Preamplifier circuit chooses amplifier with low noise and small temperature drift, otherwise noise will further enlarge by main amplifier. RC filter is used after preamplifier to filter out high frequency noise, and $\mathrm{AC}$ coupling is used to connect main amplifier to eliminate the effects of DC bias and zero drift. Filter circuit uses band-pass filter, and bandwidth is set to $8 \mathrm{KHz} \sim 12 \mathrm{KHz}$ to further reduce noise.

\subsubsection{Crude and precision fusion and error correction}

The crude and precision channel of inductosyn use AD2S80 axial angle solver chip which is tracking amplitude discrimination mode. When choosing solver chip, resolution, speed and bandwidth indexes are comprehensive considered. Crude and precision fusion algorithm based on FPGA use error larger crude channel absolute angle to find output angle cycle of precision channel and then use precise angle of precision channel to find current precise angle. The error of angle measuring system is usually system error, and repeat precision is higher than absolute precision, so this paper corrects error by broken-line approximation method to improve measuring precision of the system.

\subsection{Scanning control circuit}

Scanning control is based on position feedback control method. First of all DSP produces position command which is satisfied system motion law, and measuring circuit sends angle information into the DSP processor. In a control cycle, DSP obtain deviation of current position and predetermined position, then calculates correction torque command to drive motor. By whole digital closed loop control, disturbing torque will be effective decreased through the feedback loop and scanning linearity and control precision are effectively guaranteed ${ }^{[7]}$.

The curve of the scanning motion includes linear and reversing segments. Theoretical reversing segment motion curve should be triangular wave, but the system has first order discontinuity point.

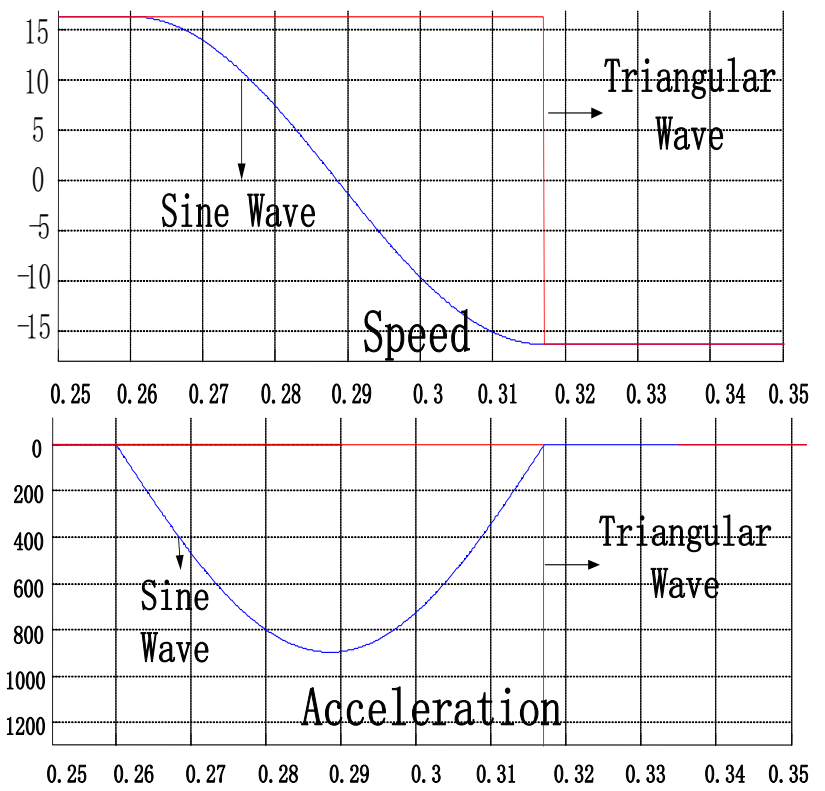

Figure 6. Comparison of speed and acceleration of sine curve and triangle curve

So sine wave is used to smoothing brake and accelerate process of reversing segment [8][9]. Velocity and acceleration of triangular wave and sine wave is showed in figure 6 . The Planning sine wave as follows:

$$
\begin{aligned}
& \theta=A \sin w t \\
& V=-A w \cos w t \\
& a=-A w^{2} \sin w t
\end{aligned}
$$

In this project, scanning frame time is $2.5 \mathrm{~s}$; scanning efficiency is $80 \%$; so nonlinear time is $0.5 \mathrm{~s}$ and sine 
period is $1 \mathrm{~S}, \mathrm{w}=2 \pi$. The torque balance relationship between scanning is:

$$
T(t)-T_{l}(t)=J a
$$

$T(t)$ is motor output torque, $T_{l}(t)$ is recovery torque of pivot (sum of two pivot), $\mathrm{J}$ is total moment of inertia of the motor coil and load, $a$ is acceleration. So when $a>0$, system is acceleration; When $a<0$, system is deceleration; when $a=0$, system is uniform.

When $t=0$, from linear segment into non-linear segment, $\theta=0, V=-A w, \mathrm{~V}$ is the scanning speed, $a=0$; so as to obtain $A=V / w$; At this time $T(t)=T_{l}(t)=2 \times$ maximum angle of linear segment $\times$ pivot tensional rigidity, so as to obtain maximum torque requirements of motor.

When $t=T / 4$, at this time scanning mirror in the maximum swing angle, $\theta=A, V=0, a=-A w^{2}$; Non-linear maximum angle and maximum system acceleration can be calculated, so as to find out the maximum disturbing torque;

When $t=T / 2$, from non-linear segment into linear segment, $\theta=0, V=A w, \quad a=0$; At the same time, $T(t)=T_{l}(t)=2$ $\times$ maximum angle of linear segment $X$ pivot tensional rigidity.

Motor output torque and disturbing torque curve is showed in figure 7 and figure 8 :

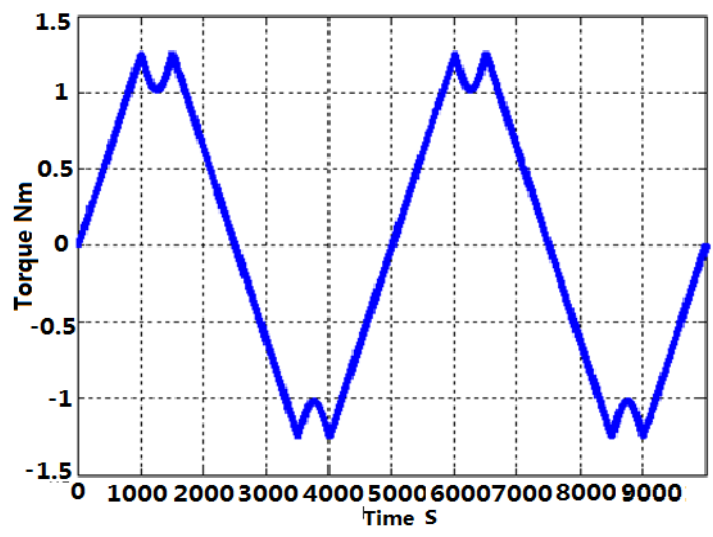

Figure 7. Motor output torque curve

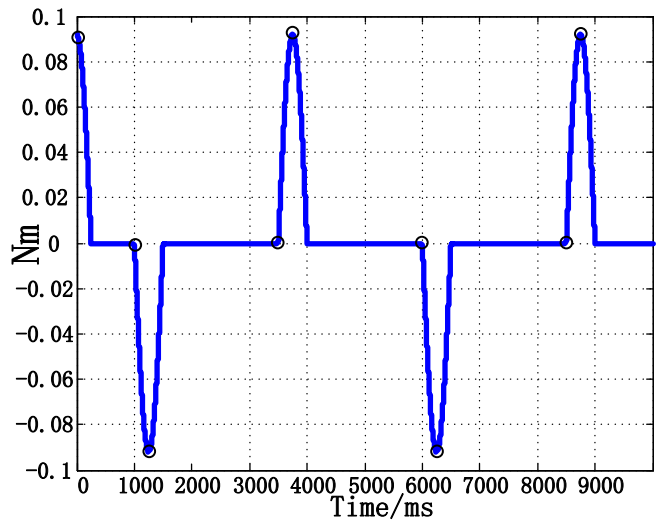

Figure 8. System disturbing torque curve

\section{Scanning precision test}

Common Angle testing methods are contact and non-contact type. Non-contact type does not need to be connected with scanning mechanism, so it will not generate disturbing torque and its precision will not influenced by scanning mechanism [10]. In this paper, the measuring precision of the scanning system requires 1 arcsecond, so precision of the test system needs to reach 0.5 arcsecond.

The non-contact measuring system is based on photoelectric autocollimator, and photoelectric autocollimator precision can reach 0.1 ". The disadvantage is that angle range is too small, so this paper uses two sets of photoelectric autocollimator and turntable combination scheme. Scanning mechanism is placed on the turntable, and plane mirror which is used for photoelectric autocollimator 1 is placed in the upper of rotating shaft. Multi-tooth dividing table is placed on turntable, and plane mirror which is used for photoelectric autocollimator 2 is placed in the upper of multi-tooth dividing table.

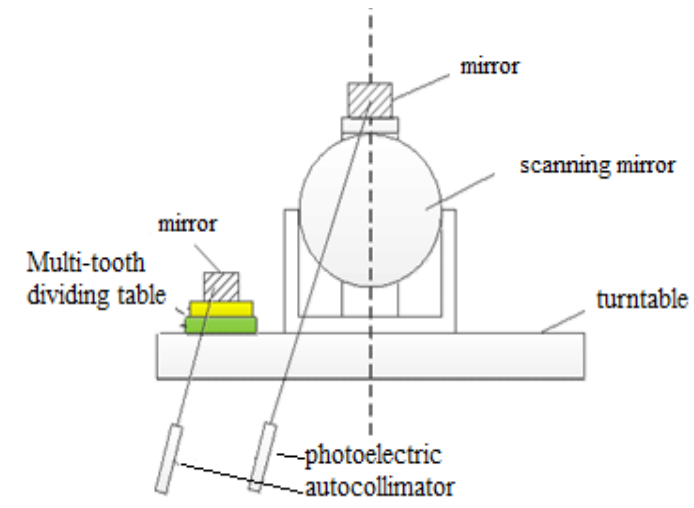

Figure 9. Principle diagram of test system

During the test, first of all two sets of photoelectric autocollimator auto collimate by mirror, then making scanning mirror rotate a certain angle, and turntable rotates the same angle in the opposite direction, so photoelectric autocollimator 1's readings backs to initial readings, this means that turntable and scanning mirror rotate the same angle. Secondly, rotating multi-tooth dividing table on the turntable, photoelectric autocollimator 2 auto collimates. The exact turn angle of turntable can be obtained by the angle of multi-tooth dividing tablee and photoelectric autocollimator 2 readings, which subtracts the angle of angle measuring circuit to obtain system angle measuring precision.

Angle measurement test results are as follows. The measuring error is less than 1 arcsecond.

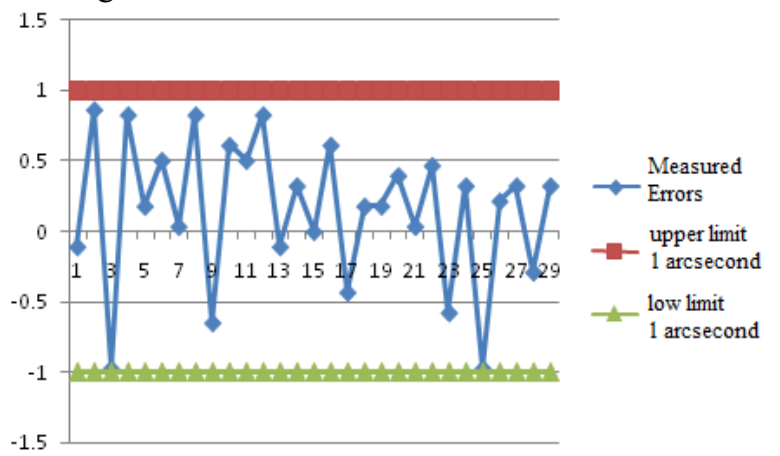

Figure 10. Angle measuring error curve 
Scanning non-linearity is the difference of angular velocity of scanning mirror at any position and average velocity divides average velocity, and scanning speed is fixed time actual output angle difference getting from angle measurement circuit divides time interval. The nonlinearity of scan test is less than $0.5 \%$. Control precision which is the maximum deviation of theory angle position and actual angle position is better than 3 arcsecond.

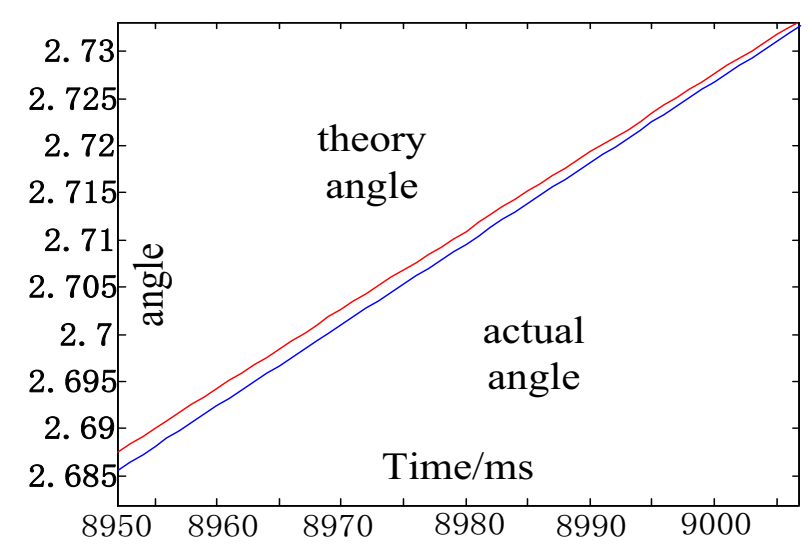

Figure 11. Scanning control precision curve

\section{Conclusions}

In this paper, the mechanism uses beryllium mirror, voice coil motor, flexible pivot and absolute inductosyn to guarantee that the system has minimum moment of inertia, minimal disturbing torque, highest angle measuring precision and high reliability. In the control aspect, angle measuring circuit and control circuit architecture of is used. Low noise angle measuring circuit ensures angle measuring precision and digital closed loop control algorithm guarantees that the system has sufficient servo stiffness. Scanning reversing segment uses sine wave, and system disturbing torque, motor output torque and reversing angle calculation method is given. Finally, a non-contact measuring method proves that system angle measuring precision can reach 1 arcsecond, non-linearity can be less than $0.5 \%$, control precision is better than 3 arcsecond.

\section{References}

1. Yuan L, Yang L, "Research on low speed, big Inertia and high precision scanning mechanism of satellite camera,"The twelfth session of the National Conference on space and motor control technology, 2000.

2. Sun D, "Optimum design and experimental study of flexible support system of directed structure," Harbin Institute of Technology,2009.

3. Qin H, Wang P, "Research on high accuracy swing scanning motor and control system,"The twelfth session of the National Conference on space and motor control technology,2000.

4. ZHANG D,FENG X. "The Technical Principle of Voice-Coil Actuator," Journal of north university of china, Papers 27(3), 224-228 (2006).

5. Huo S, "Round inductosyn turntabl eangular measuring system based on dsp and cpld," Harbin Institute of Technology,2009 .

6. QIU Z,LI W,XU B, "Absolute Inductosyn Angle Measurement System Based on DSP and AD2S80 ,'Instrument Technique and Sensor, Papers 8(8), 59-62 (2010).

7. Cai Q, "The Research and Design of Scanning Control Systembased on DSP," Shanghai Jiao Tong University, 2008.

8. Luo Q,Yuan L,Yang L. "A method for Driving Scan Mirror," Spacecraft Recovery \& Remote Sensing, Papers 27(1), 36-41 (2006).

9. Wu Q,Wang Q,Peng Q, "Wide bandwidth control of fast-steering mirror driven by voice coil motor," Opto-Electronic Engineering, Papers 31(8), 15-18 (2004).

10. YANG X,ZHANG T,ZHANG J, "Design of a system for testing scanner's motion control characteristics," Electronics Optics \& Control, Papers 19(8), 78-81 (2012). 